\title{
Fabrication and characterization of multiscale electrospun scaffolds for cartilage regeneration
}

\author{
Erica J Levorson ${ }^{1}$, Perumcherry Raman Sreerekha ${ }^{2}$, \\ Krishna Prasad Chennazhi ${ }^{2}$, F Kurtis Kasper ${ }^{1}$, Shantikumar V Nair ${ }^{2,4}$ \\ and Antonios G Mikos ${ }^{1,3,4}$ \\ ${ }^{1}$ Department of Bioengineering, Rice University, MS-142, PO Box 1892, Houston, TX 77251-1892, \\ USA \\ 2 Amrita Center for Nanosciences and Molecular Medicine, Amrita Institute of Medical Sciences and \\ Research Centre, Cochin-682 041, India \\ ${ }^{3}$ Department of Chemical and Biomolecular Engineering, Rice University, MS-362, PO Box 1892, \\ Houston, TX 77251-1892, USA \\ E-mail: shantinair@aims.amrita.edu and mikos@rice.edu
}

Received 9 February 2012

Accepted for publication 18 June 2012

Published 25 January 2013

Online at stacks.iop.org/BMM/8/014103

\begin{abstract}
Recently, scaffolds for tissue regeneration purposes have been observed to utilize nanoscale features in an effort to reap the cellular benefits of scaffold features resembling extracellular matrix (ECM) components. However, one complication surrounding electrospun nanofibers is limited cellular infiltration. One method to ameliorate this negative effect is by incorporating nanofibers into microfibrous scaffolds. This study shows that it is feasible to fabricate electrospun scaffolds containing two differently scaled fibers interspersed evenly throughout the entire construct as well as scaffolds containing fibers composed of two discrete materials, specifically fibrin and poly( $\varepsilon$-caprolactone). In order to accomplish this, multiscale fibrous scaffolds of different compositions were generated using a dual extrusion electrospinning setup with a rotating mandrel. These scaffolds were then characterized for fiber diameter, porosity and pore size and seeded with human mesenchymal stem cells to assess the influence of scaffold architecture and composition on cellular responses as determined by cellularity, histology and glycosaminoglycan (GAG) content. Analysis revealed that nanofibers within a microfiber mesh function to maintain scaffold cellularity under serum-free conditions as well as aid the deposition of GAGs. This supports the hypothesis that scaffolds with constituents more closely resembling native ECM components may be beneficial for cartilage regeneration.

(Some figures may appear in colour only in the online journal)
\end{abstract}

\section{Introduction}

Fibrous scaffolds are commonly used in tissue engineering due to their characteristic high porosities and interconnected pores, which have been shown to facilitate cellular infiltration and homogenous tissue regeneration [1-3]. Previous reports have indicated that the size scale of the fibers in such a

\footnotetext{
${ }^{4}$ Authors to whom any correspondence should be addressed.
}

scaffold can have a significant effect on tissue development. Specifically, nanoscale features are desired because they more closely resemble components of a native extracellular matrix (ECM) such as collagen fibers [4-6]. However, it is also fairly well accepted that high concentrations of nanoscale fibers often lead to increased cell spreading and limited cellular infiltration $[7,8]$. For this reason, the logical progression is to fabricate scaffolds that combine microfibers with nanofibers, in an effort to maintain larger pore sizes, while harnessing the positive 


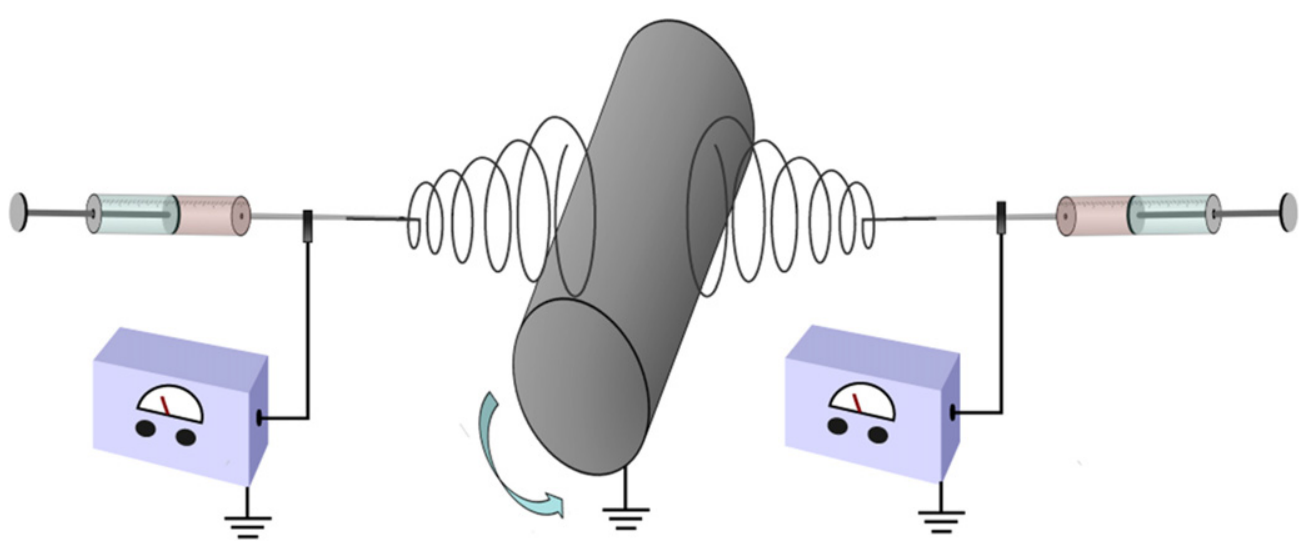

Figure 1. Dual extrusion electrospinning with a rotating mandrel diagram.

effects of nanofibers, such as increased cellular attachment as well as improved differentiation and ECM production [9]. While previous efforts have combined nanofibers with microfibers to form multiscale scaffolds in a layer-by-layer electrospinning approach or by combining electrospinning with three-dimensional melt deposition, few efforts report the fabrication of a completely electrospun multiscale scaffold generated by continuous means with a relatively homogenous distribution of micro and nanofibers throughout the entire scaffold [7, 8, 10-13]. Soliman et al describe a method whereby multiscale scaffolds are formed by dual extrusion of fibers onto a flat stage pivoting orthogonally to the collection surface to facilitate fiber mixing [14]. An alternative fiber mixing strategy is demonstrated in this work in which a cylindrical rotating mandrel is used to collect the electrospun fibers.

With the notion of creating scaffolds of varied internal structures or size scales comes the concept of altering the material composition in an effort to exact a more favorable cellular response. As in any field of tissue engineering, both natural and synthetic materials are being explored for generating engineered cartilage. Natural biomaterials for cartilage engineering tend to be either protein, such as collagen and fibrin [15-18], or polysaccharide based, such as alginate and chitosan [19-24]. The benefit of using natural materials is that they innately support cell attachment and proliferation and are generally biodegradable. Alternatively, the properties of synthetic materials are easily reproducible and tunable with known degradation rates and low immunogenicity. A scaffold which is able to combine fibers of a synthetic polymer to harness its reproducible properties with some fibers of a natural composition to improve cellular responses could be quite advantageous. Recently, it was confirmed that fibrin may be electrospun into nanofibrous structures [25]. This offers a unique opportunity to generate a completely electrospun composite fibrous scaffold by uninterrupted methods containing differently scaled fibers composed of two separate materials, specifically, a highly bioactive natural material such as fibrin and a synthetic material such as poly( $\varepsilon$-caprolactone) (PCL).

The objective of this study was to demonstrate the feasibility of fabricating electrospun scaffolds consisting of two differently scaled fibers interspersed evenly throughout an entire construct as well as scaffolds containing fibers composed of two individual materials, specifically fibrin and PCL. These different scaffold types were then characterized and examined to determine whether the presence of nanofibers, either synthetic or natural, within a mesh of microfibers had any effect on cellularity and glycosaminoglycan (GAG) deposition over scaffolds composed solely of microfibers. It was hypothesized that scaffolds containing bioactive nanofibers composed of fibrin would lead to improved cellular proliferation, distribution and GAG production when compared to scaffolds composed entirely of PCL regardless of scaffold architecture.

\section{Methods}

\subsection{Electrospinning}

Scaffolds containing PCL microfibers $(\mathrm{P} \mu)$, PCL microfibers with PCL nanofibers ( $\mathrm{P} \mu \mathrm{Pn})$, and PCL microfibers and fibrin nanofibers $(\mathrm{P} \mu \mathrm{Fn})$ were electrospun using a dual extrusion process with a rotating mandrel to ensure thorough mixing of the two fiber types (figure 1). The mandrel $(\varnothing=4.8 \mathrm{~cm})$ was set to rotate at a maximum speed of 120 RPM to facilitate fiber mixing without resulting in aligned fibers. Microfibers were fabricated using a 35\% (w/v) PCL (MW = 43000-50 000, Polysciences, Warrington, PA) solution in a $5: 1(\mathrm{v} / \mathrm{v})$ ratio of chloroform: methanol extruded at a rate of $8 \mathrm{~mL} \mathrm{~h}^{-1}$ with $7 \mathrm{kV}$ applied voltage. Prior to electrospinning, fibrinogen was isolated from human plasma by cryoprecipitation as previously described [25, 26]. Fibrin nanofibers were generated using previously described methods [25]. Specifically, $50 \mathrm{mg}$ $\mathrm{mL}^{-1}$ fibrinogen in $6 \%(\mathrm{w} / \mathrm{v})$ poly(vinyl alcohol) (PVA) and $300 \mathrm{U}$ thrombin (Merck, Darmstadt, Germany) in $8 \%$ (w/v) PVA with $10 \mathrm{mM}$ calcium chloride were co-extruded from separate syringes through a mixing applicator tip at a rate of $0.4 \mathrm{~mL} \mathrm{~h}^{-1}$ with an applied voltage of $18 \mathrm{kV}$. PCL nanofibers were fabricated using a $32 \%(\mathrm{w} / \mathrm{v})$ PCL solution in formic acid extruded at a rate of $0.1 \mathrm{~mL} \mathrm{~h}^{-1}$ with a $13 \mathrm{kV}$ applied voltage. Prior to sterilization for cell seeding, meshes containing PCL nanofibers were washed thoroughly with phosphate buffered saline (PBS) and air dried for one day to remove any residual formic acid. 


\subsection{Scaffold morphology}

Electrospun scaffolds were mounted on aluminum stubs and sputter-coated with $20 \mathrm{~nm}$ of gold prior to imaging with a scanning electron microscope (SEM) (FEI Quanta 400 ESEM FEG, FEI Co, Hillsboro, OR). The average microfiber diameter for each scaffold type was determined from six separate SEM images taken at $1000 \times$ magnification from three random locations on the electrospun mat. On each SEM image, the diameter of five fibers was measured using the manufacturer supplied software for a total of 30 discrete measurements per scaffold type. Nanofibers were measured from SEM images of three random locations on the electrospun mat taken at $15000 \times$ with ten fibers measured per location for a total of 30 separate measurements. Furthermore, two discs $(\varnothing=$ $6 \mathrm{~mm}$ ) from different locations on each type of fibrous mat generated were taken and prepared for SEM imaging of a scaffold vertical cross-section. To accomplish this, each die cut scaffold was submerged in liquid nitrogen for $1 \mathrm{~min}$ to aid in achieving a clean vertical cross-section. After the scaffolds were thoroughly frozen, they were sectioned perpendicular to the scaffold face into thin slices using a microtome blade, mounted on aluminum stubs, sputter-coated, and imaged via SEM.

\subsection{Scaffold porosity}

The porosity and pore size of the electrospun meshes were determined via mercury intrusion porosimetry. First, the bulk volume and weight of each scaffold were measured. Scaffolds were then placed individually in a mercury porosimeter (Quantachrome Instruments, Boynton Beach, FL) which forced mercury through the pores of the scaffolds $(n=3)$ ultimately reaching a final pressure of $50 \mathrm{psi}$. For the contact angle between PCL and mercury in air, an angle of $140^{\circ}$ was used as reported in previous work [7]. The porosity $(\varepsilon)$ was then calculated as

$$
\varepsilon=\frac{V_{\text {pore }}}{V_{\text {pore }}+1 / \rho} \times 100 \%,
$$

in which the pore volume $\left(V_{\text {pore }}\right)$ was estimated as the volume of intruded mercury per gram of scaffold and $\rho$ is the density of PCL. The pore size was computed from the volume versus pressure data using the manufacturer supplied software (Quantachrome Poremaster for Windows, version 8). For mercury porosimetry calculations regarding the $\mathrm{P} \mu \mathrm{Fn}$ scaffolds, it was assumed that the effect of fibrin on the bulk material density was negligible. Thus for these scaffolds the bulk material density was assumed to be equal to that of PCL alone.

\subsection{Cell isolation and expansion}

Human mesenchymal stem cells (hMSCs) were isolated from umbilical cord blood using a Ficoll-Paque gradient (Amersham-Pharmacia), plastic adherence selection and flow cytometry verification of the MSC phenotype as previously described [25]. Specifically, the cell population isolated with flow cytometry displayed positive expression of surface markers CD29, CD44, CD73 and CD90 and negative expression of markers CD31, CD33, CD34 and CD45 which are often used to indicate the MSC phenotype [27-30]. Similarly sourced cells have been previously shown to successfully differentiate toward the osteoblastic and chondrocytic lineages [29-32]. After isolation, cells were expanded in general growth medium containing Iscove's Modified Dulbecco's Medium (Gibco), 10\% MSC-qualified fetal bovine serum (FBS) (Gibco), 10\% general FBS (Gibco), as well as $1 \%$ penicillin-streptomycin.

\subsection{Cell seeding and culture}

Electrospun scaffolds were die cut into discs of $6.2 \mathrm{~mm}$ in diameter and ethylene oxide sterilized. Only scaffolds 0.4$0.6 \mathrm{~mm}$ thick were used for cell studies. Prior to seeding, scaffolds were sterilely pre-wet using a decreasing ethanol gradient (90-35\%), rinsed with sterile water and centrifuged, and then incubated in general growth medium overnight. The pre-wet scaffolds were then pressfit into 96 well plates and seeded with 75000 passage 6 hMSCs per scaffold. Seeded scaffolds were then incubated for a $3 \mathrm{~h}$ pre-attachment period. After the pre-attachment period, the wells were filled with chondrogenic medium containing Dulbecco's Modified Eagle's Medium (Gibco), $1 \% \mathrm{ITS}^{+}$premix (BD Biosciences), $50 \mathrm{mg} \mathrm{L}^{-1}$ ascorbic acid, $10^{-7} \mathrm{M}$ dexamethasone and $0.4 \mathrm{mM}$ L-proline, and incubated for the remaining $24 \mathrm{~h}$ attachment period. Day 0 samples were prepared for analysis after the $3 \mathrm{~h}$ pre-attachment period. After the $24 \mathrm{~h}$ attachment period, the scaffolds were unconfined from the 96 well plates and placed in 24 well plates with chondrogenic medium for the duration of the study. Medium was changed every three days. Samples were taken at days $0,7,14$, and 21, washed with PBS $3 \times$, and prepared for analysis.

\subsection{Construct imaging}

For visualization via SEM, cultured constructs were fixed by incubating with $2 \%$ glutaraldehyde for $1 \mathrm{~h}$. Samples were then dehydrated using an increasing ethanol gradient (30-90\%) and dried. The samples $(n=2)$ were then sputter-coated with $20 \mathrm{~nm}$ gold and imaged using SEM (JSM-6490LA, Tokyo, Japan) to visualize cell spreading and ECM production on the surface of the different scaffold types.

In preparation for confocal imaging, samples $(n=2)$ were fixed in $4 \%$ paraformaldehyde for $20 \mathrm{~min}$ and then rinsed with PBS. Cell membranes were permeabilized with $0.5 \%$ Triton X-100 in PBS for 10 min and then washed with $1 \%$ FBS and PBS to minimize background staining. Finally, the cells were stained for F-actin with 1:1000 Texas Red-X phalloidin (molecular probes) in PBS for $1 \mathrm{~h}$. Samples were rinsed with PBS to remove any unconjugated probes. The stained scaffolds were imaged at 594 and $488 \mathrm{~nm}$ wavelengths and $20 \times$ magnification using a confocal microscope (Leica TCS SP5 II). In addition to composite z-stack images, image slices were viewed separately in succession to examine individual cellular morphologies. 


\subsection{Histology}

At each time point, samples $(n=2)$ were fixed in $10 \%$ buffered paraformaldehyde overnight at $4{ }^{\circ} \mathrm{C}$. Scaffolds were then dehydrated using an increasing ethanol gradient (30$90 \%$ ) and stored in $100 \%$ isopropanol at $4{ }^{\circ} \mathrm{C}$. Prior to sectioning, samples were embedded in HistoPrep frozen tissue embedding medium (Fisher Scientific, Pittsburgh, PA). A cryotome (CM1850, Leica Microsystems, Bannockburn, IL) was used to cut sections $8 \mu \mathrm{m}$ thick which were then mounted on glass slides and incubated on a slide warmer at $45^{\circ} \mathrm{C}$ for a minimum of five days.

To visualize the cartilage-like ECM produced, sections were rehydrated with water and then stained with $0.1 \%$ Safranin $O$ for $5 \mathrm{~min}$. The slides were then rinsed with water and air dried. Stained sections were imaged with a light microscope (Zeiss AxioImager.Z2, Göttingen, Germany) using a peripheral camera (AxioCam MRc 5, Zeiss, Göttingen, Germany) and computer. Automatic exposure and white balance correction were applied to the captured images using the manufacturer supplied software (AxioVision 4.8, Zeiss, Göttingen, Germany).

In order to visualize cellular infiltration of the different scaffold architectures, sections were also stained with Fast Green FCF (Sigma). Sections were first washed with $1 \%$ acetic acid and then treated with $0.02 \%$ Fast Green FCF for 3 min at which point the slides were then rinsed with $1 \%$ acetic acid. The stained sections were imaged using a light microscope with automatic exposure and white balance correction applied using the manufacturer supplied software.

\subsection{Biochemical assays}

In an effort to determine the cellularity and GAG content of the scaffolds at the various time points, samples $(n=4)$ were initially placed in $0.5 \mathrm{~mL}$ of water and frozen at $-80{ }^{\circ} \mathrm{C}$. The samples were then treated with three freeze/thaw/sonicate cycles resulting in a cell lysate. The DNA content of the cell lysate was then quantified using the fluorometric PicoGreen assay kit (Molecular Probes, Eugene, OR) with an excitation wavelength of $490 \mathrm{~nm}$ and an emission wavelength of $520 \mathrm{~nm}$. The dsDNA standards and samples were run side by side in triplicate to calculate the DNA content per scaffold.

Prior to quantifying GAG content, scaffolds were removed from the cell lysate used in determining DNA content and digested at $56{ }^{\circ} \mathrm{C}$ in $0.5 \mathrm{~mL}$ of proteinase $\mathrm{K}$ $\left(1 \mathrm{mg} \mathrm{mL} \mathrm{m}^{-1}\right.$ proteinase $\mathrm{K}, 185 \mu \mathrm{g} \mathrm{mL}^{-1}$ iodacetamide and $10 \mu \mathrm{g} \mathrm{mL}^{-1}$ pepstatin $\mathrm{A}$ in Tris/EDTA buffer containing $6.055 \mathrm{mg} \mathrm{mL}^{-1}$ tris(hydroxymethyl aminomethane and $372 \mu \mathrm{g}$ $\mathrm{mL}^{-1}$ EDTA at $\mathrm{pH}$ 7.6). GAG content was then quantified using the 1,9-dimethymethylene blue (DMMB) assay and measured at $520 \mathrm{~nm}$ using a microplate reader (BIO-TEK Instrument, Winooski, VT). Chondroitin sulfate standards, cell lysate samples and proteinase $\mathrm{K}$ digest samples were all run in duplicate and then averaged to adjust for any experimental error. GAG concentrations determined from the cell lysate and proteinase $\mathrm{K}$ digest for each individual sample were summed to determine the final GAG content for each sample.
Table 1. Scaffold characteristics including average micro and nanofiber diameters, porosity and pore size. Within a particular characteristic, a scaffold type which is determined to be statistically different from both of the other scaffold types is indicated by an asterisk, '*' $(p<0.05)$.

\begin{tabular}{lccc}
\hline Characteristic & \multicolumn{1}{c}{$\mathrm{P} \mu$} & $\mathrm{P} \mu \mathrm{Pn}$ & $\mathrm{P} \mu \mathrm{Fn}$ \\
\hline $\begin{array}{l}\text { Microfiber } \\
\text { diameter }(\mu \mathrm{m})\end{array}$ & $10.1 \pm 1.1$ & $9.8 \pm 1.4$ & $8.8 \pm 1.1^{*}$ \\
$\begin{array}{l}\text { Nanofiber } \\
\text { diameter }(\mathrm{nm})\end{array}$ & $0 \pm 0^{*}$ & $194.6 \pm 45.9^{*}$ & $250.0 \pm 50.2^{*}$ \\
$\begin{array}{l}\text { Porosity }(\%) \\
\text { Pore size }(\mu \mathrm{m})\end{array}$ & $32.5 \pm 0.4^{*}$ & $91.8 \pm 1.7$ & $90.0 \pm 0.7$ \\
\hline
\end{tabular}

\subsection{Statistical methods}

Scaffold characteristics and biochemical assay results are reported as means \pm standard deviation. A one-way ANOVA was performed to evaluate significant differences $(p<0.05)$ in fiber diameter, porosity and pore size. A two-way ANOVA was performed to evaluate significant differences $(p<0.05)$ in construct cellularity and ECM content. If significant difference was determined, the Tukey's HSD test was performed to make multiple comparisons between groups.

\section{Results}

\subsection{Scaffold characterization}

SEM images of the different scaffold types demonstrated that it was possible to fabricate electrospun mats using continuous methods to create scaffolds of two different fiber scales as well as two different materials (figure 2). Images of vertical scaffold cross-sections taken using SEM revealed that when nanofibers were incorporated into a microfiber mesh using the dual extrusion electrospinning process with a rotating mandrel, nanofibers were truly interspersed throughout the entire construct (figures 2( $g$ )- $(i)$ ). These images also showed that, with respect to volume, the nanofiber containing scaffolds were composed of more microfibers than nanofibers due to the much lower polymer extrusion rates necessary to produce nanofibers compared to microfibers. The scaffolds that were generated consisted of fairly similarly sized microfibers with averages between 8.8 and $10.1 \mu \mathrm{m}$. It is important to note, however, that the $\mathrm{P} \mu \mathrm{Fn}$ scaffold fiber diameter (8.8 \pm $1.1 \mu \mathrm{m})$ was significantly lower than the other two scaffold types (table 1). Despite having a smaller average microfiber diameter, the $\mathrm{P} \mu \mathrm{Fn}$ scaffolds still displayed porosities similar to $\mathrm{P} \mu \mathrm{Pn}$ scaffolds as well as significantly higher porosities than microfibers alone. Examining the nanofibers in the $\mathrm{P} \mu \mathrm{Pn}$ and $\mathrm{P} \mu$ Fn scaffolds, it was observed that nanofibers, regardless of composition, exhibited diameters that were primarily less than $400 \mathrm{~nm}$ when using these methods of fabrication. Furthermore, it was evident that the $\mathrm{P} \mu \mathrm{Pn}$ scaffolds contained a higher density of nanofibers than the $\mathrm{P} \mu \mathrm{Fn}$ scaffolds due to the different extrusion rates necessary to stably electrospin the differently composed nanofibers.

Porosity as determined by mercury intrusion demonstrated that the scaffolds exhibited reasonably high porosities even with the incorporation of nanofibers. 

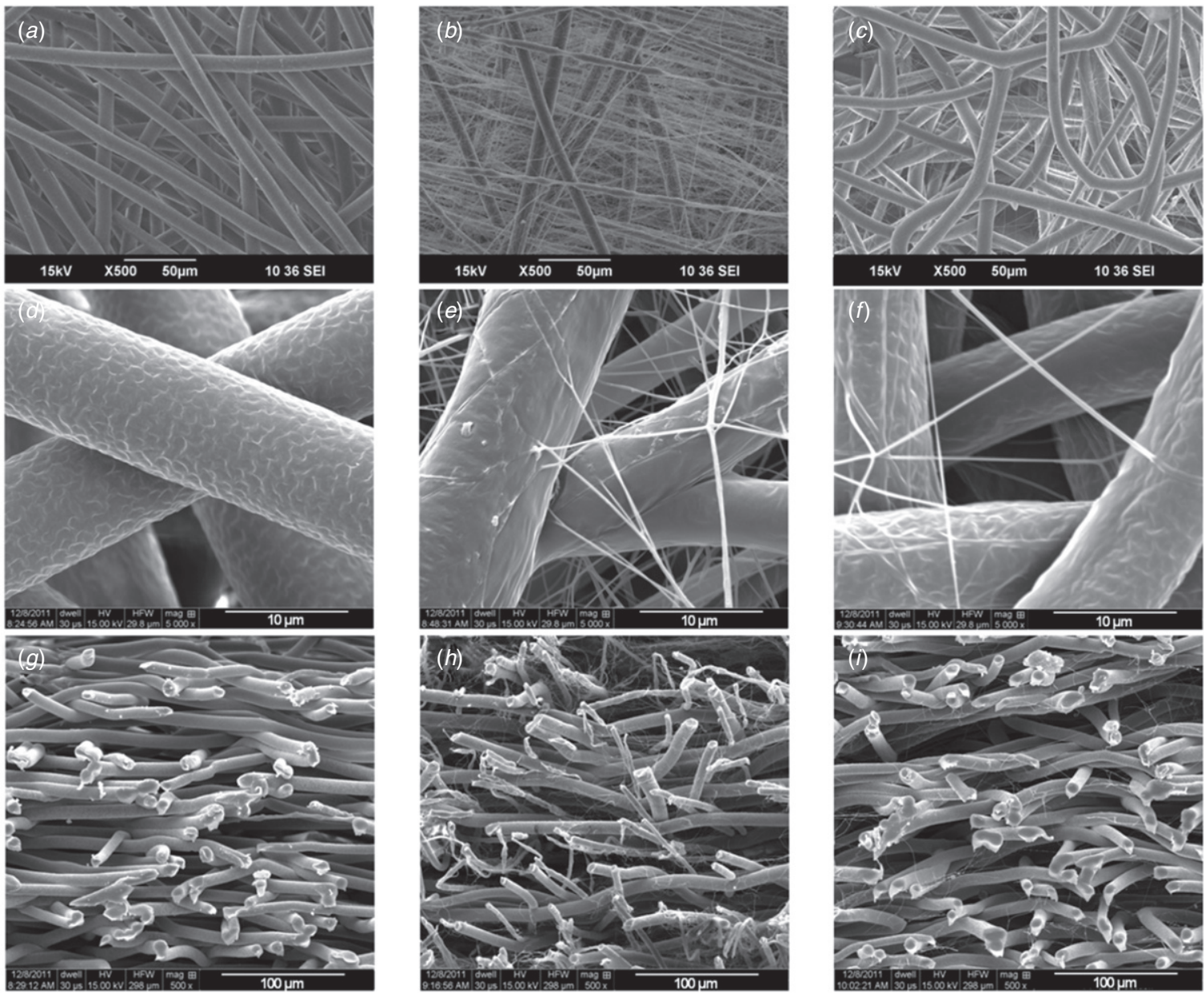

Figure 2. Scanning electron micrograph images of the different electrospun scaffold architectures and compositions that were generated: (a), (d) $\mathrm{P} \mu,(b),(e) \mathrm{P} \mu \mathrm{Pn},(c),(f) \mathrm{P} \mu \mathrm{Fn}$. Vertical cross-sections of the different scaffolds show the distribution of nanofibers with respect to microfibers throughout the thickness of the scaffold: $(g) \mathrm{P} \mu,(h) \mathrm{P} \mu \mathrm{Pn},(i) \mathrm{P} \mu \mathrm{Fn}$.

In fact, the $\mathrm{P} \mu$ scaffolds had a significantly lower porosity than the other two scaffold groups which featured nanofibers. Despite displaying a lower porosity than the other two scaffold types, the $\mathrm{P} \mu$ scaffolds bore significantly larger pore sizes than the scaffolds containing nanofibers. Additionally, the $\mathrm{P} \mu \mathrm{Pn}$ scaffolds, which had the highest density of nanofibers as seen in SEM images, exhibited the smallest average pore size of all of the scaffold types.

\subsection{Construct imaging}

From confocal images, it was seen that after only $3 \mathrm{~h}$, cell spreading was evident on all three scaffold types (figure 3 ). However, on the $\mathrm{P} \mu \mathrm{Pn}$ scaffolds cells appeared to display a flattened, broad polygonal morphology, while cells on the $\mathrm{P} \mu$ and $\mathrm{P} \mu \mathrm{Fn}$ scaffolds exhibited more elongated and spindle-like morphologies, as indicated with arrows included in figure 3. Likewise, hMSCs on the $\mathrm{P} \mu \mathrm{Pn}$ scaffolds displayed a different pattern of cell localization as compared to the other two scaffold types. In this case, cells on $\mathrm{P} \mu$ and $\mathrm{P} \mu \mathrm{Fn}$ scaffolds were primarily attached and spread along microfibers, while the cells on the $\mathrm{P} \mu \mathrm{Pn}$ scaffolds appeared to span the area between the microfibers more often than on the other two scaffold types.
Scanning electron micrographs revealed that throughout the entire culture period cells and ECM appeared denser on the surface of scaffolds containing PCL nanofibers (figure 4). Similarly, early in the culture duration scaffolds containing nanofibers, $\mathrm{P} \mu \mathrm{Pn}$ and $\mathrm{P} \mu \mathrm{Fn}$, displayed more cells and ECM on the scaffold surface. At a later culture duration, it was observed that fewer cells and ECM were located on the surface of the $\mathrm{P} \mu$ and $\mathrm{P} \mu \mathrm{Fn}$ scaffolds, while the surface of the $\mathrm{P} \mu \mathrm{Pn}$ scaffolds was almost completely coated with cells and ECM.

\subsection{Histology}

In an effort to examine the production and deposition of ECM within the different scaffolds as well as cellular infiltration, samples from each time point were cryosectioned and stained with either Safranin O for GAGs (figure 5) or Fast Green for cytoplasm (figure 6). Looking at Safranin O staining, negligible staining was seen at day 0 as expected. However as time progressed, scaffolds containing nanofibers $(\mathrm{P} \mu \mathrm{Pn}$ and $\mathrm{P} \mu \mathrm{Fn}$ ) exhibited more deposition of GAGs than scaffolds composed of microfibers alone $(\mathrm{P} \mu)$ as was seen on the day 21 histological sections in figure 5. On the $\mathrm{P} \mu \mathrm{Fn}$ scaffolds, GAG deposits were seen deep within the interior of the scaffold in addition to the coating on the surface. These outcomes confirm the result of the DMMB biochemical assay for GAG 

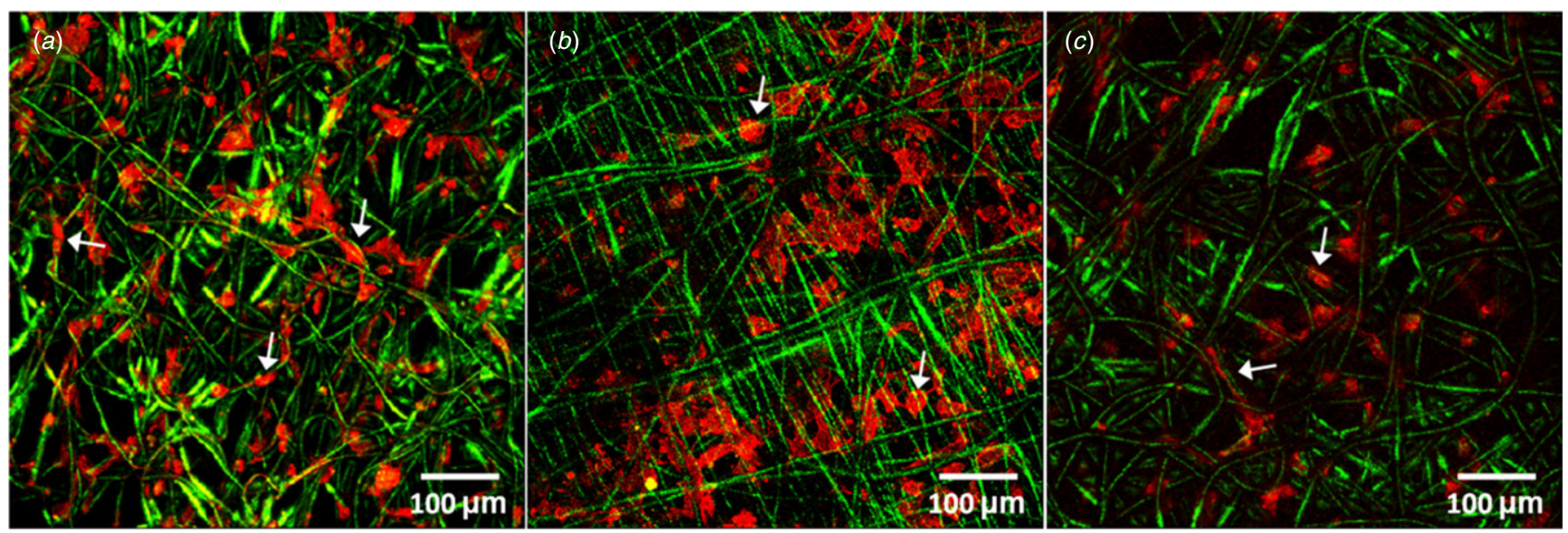

Figure 3. Composite z-stack confocal images showing spreading and arrangement of phalloidin labeled cells highlighting F-actin (red) on day 0 (a) $\mathrm{P} \mu$ (b) $\mathrm{P} \mu \mathrm{Pn}$ and (c) $\mathrm{P} \mu \mathrm{Fn}$ scaffolds (green). Arrows indicate examples of individual cell morphologies and arrangements.
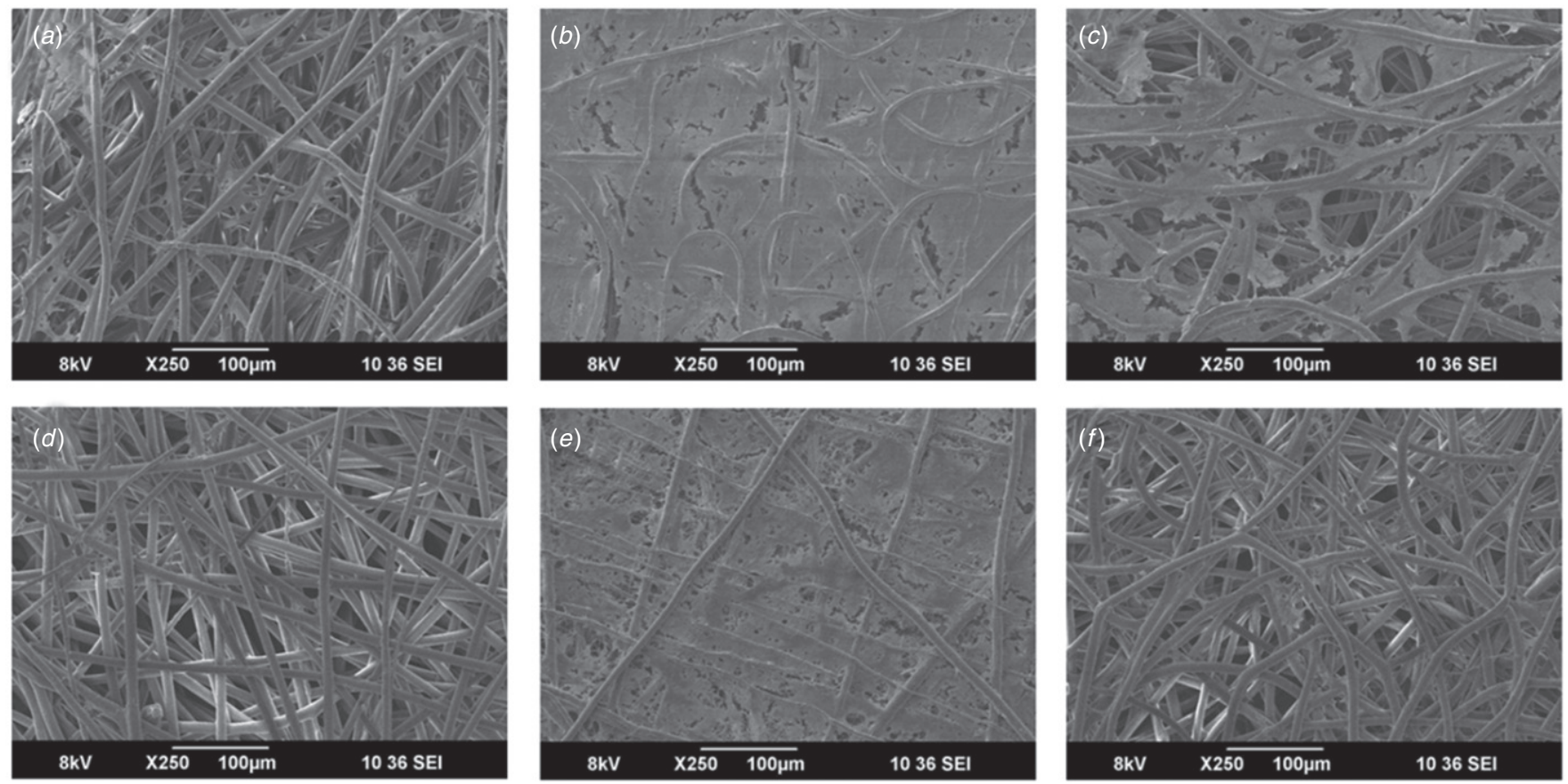

Figure 4. SEM images of constructs showing cells and ECM deposition on the surface of $(a),(d) \mathrm{P} \mu,(b),(e) \mathrm{P} \mu \mathrm{Pn}$, and $(c),(f) \mathrm{P} \mu \mathrm{Fn}$ scaffolds on $(a),(b),(c)$ day 7 and $(d),(e),(f)$ day 21.

content. With respect to cellular infiltration as viewed by Fast Green staining, it appeared that maximum cellular depth was fairly similar in all three scaffold types (figure 6). In the uppermost portion of the scaffolds, the $\mathrm{P} \mu \mathrm{Pn}$ scaffolds displayed a more scattered distribution of cells while cells were primarily located on the surface of the other two scaffold types. However, while images showed a higher occurrence of Fast Green staining in the upper half of the $\mathrm{P} \mu \mathrm{Pn}$ scaffolds, the $\mathrm{P} \mu$ and $\mathrm{P} \mu \mathrm{Fn}$ scaffolds appeared to exhibit greater Fast Green staining at the ultimate depth of infiltration than was observed in the $\mathrm{P} \mu \mathrm{Pn}$ group.

\subsection{Biochemical assays}

None of the scaffold types displayed a significant increase or decrease in total DNA content throughout time with respect to the initial cell seeding suspension (figure 7). This demonstrated that cell numbers remained fairly constant throughout the duration of the study regardless of scaffold composition and architecture.

To assess the effect of scaffold architecture on the production of GAGs, a common cartilaginous ECM component, by hMSCs, the DMMB biochemical assay for sulfated GAGs was performed. At day 14 , the $\mathrm{P} \mu \mathrm{Fn}$ samples displayed significantly higher GAG contents $(0.87 \pm 0.2 \mu \mathrm{g}$ per scaffold) than both of the other scaffold types at the various time points with exception to the $\mathrm{P} \mu \mathrm{Pn}$ samples on day $14(0.46 \pm 0.2 \mu \mathrm{g}$ per scaffold) (figure 8). No significant difference was observed in GAG deposition with respect to time for any of the three scaffold types. When GAG content was normalized to DNA content, trends appeared similar to those of the GAG data that was not normalized (figure 9). 


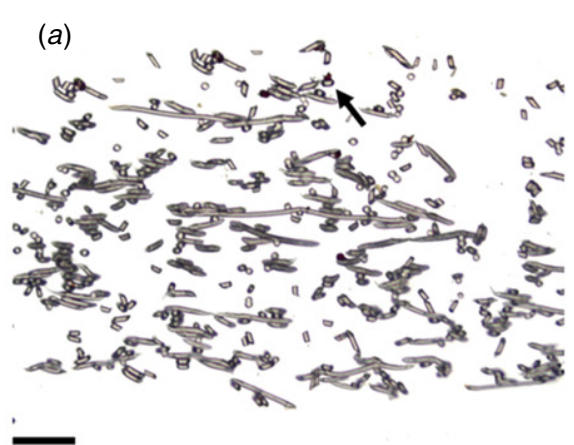

(b)

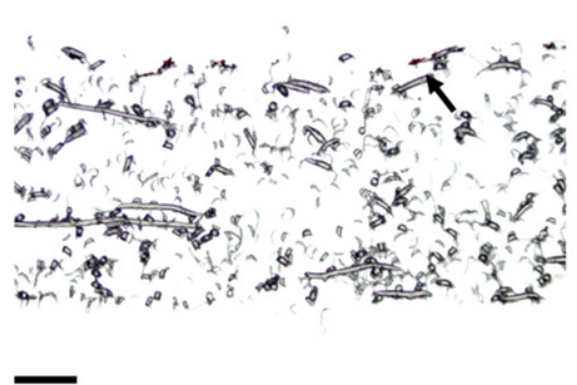

(e)

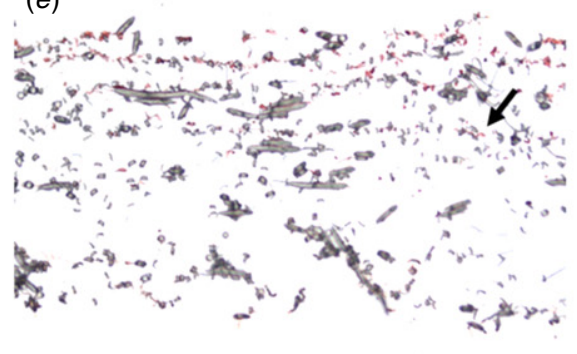

(c)
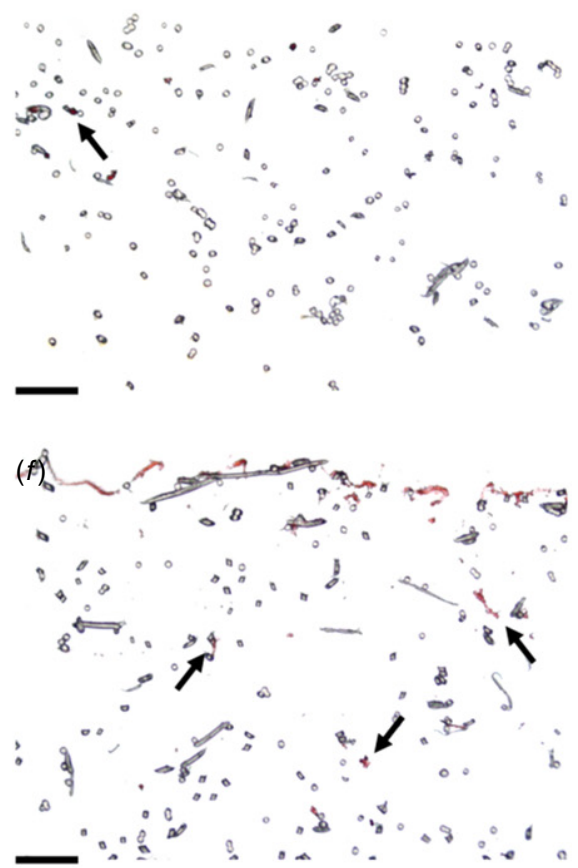

Figure 5. Safranin O staining for GAGs of $(a),(b),(c)$ day 0 and $(d),(e),(f)$ day 21 histological sections for $(a),(d) \mathrm{P} \mu,(b),(e) \mathrm{P} \mu \mathrm{Pn}$, and $(c),(f) \mathrm{P} \mu \mathrm{Fn}$ scaffolds. Arrows indicate regions of staining within the interior of the construct. Scale bars represent $100 \mu \mathrm{m}$.
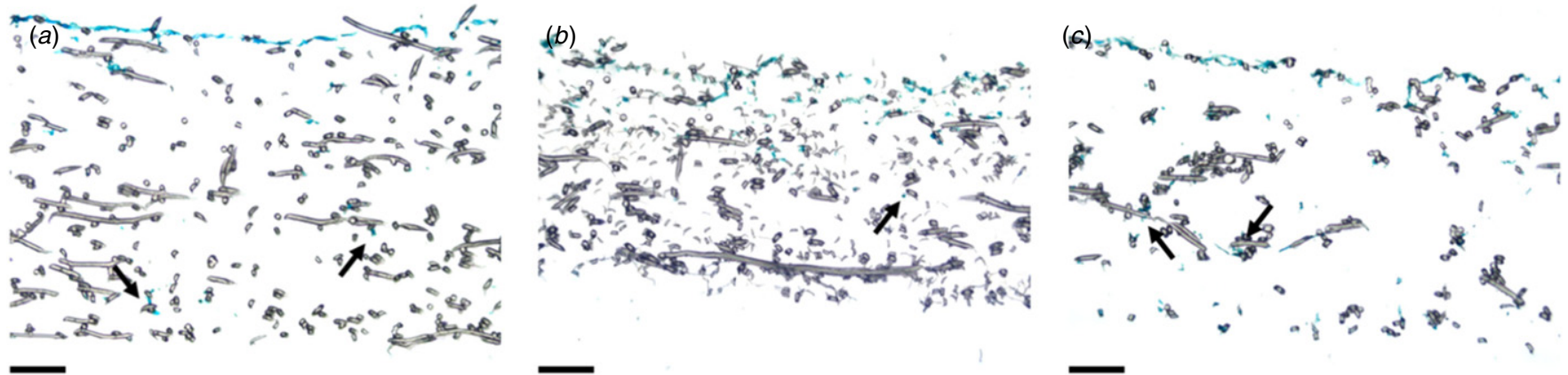

Figure 6. Fast Green staining for cytoplasm of day 21 histological sections for (a) $\mathrm{P} \mu$ day $21,(b) \mathrm{P} \mu \mathrm{Pn}$ day 21 , and (c) $\mathrm{P} \mu \mathrm{Fn}$ day 21 scaffolds. Arrows indicate regions of staining within the interior of the construct. Scale bars represent $100 \mu \mathrm{m}$.

However, there was no statistically significant difference in normalized GAG contents among the different scaffold types. Similarly, there was no statistical difference in GAG/DNA amounts with time for any scaffold type.

\section{Discussion}

In order for tissue engineering to progress, it is becoming increasingly important to develop materials that function beyond mere support structures for cells. Previous work has shown that electrospun PCL scaffolds support the attachment and deposition of cartilage-like ECM by chondrocytes and MSCs possibly because the three-dimensional architecture of electrospun PCL resembles the morphology of collagenous ECM [5, 33]. As such, it follows that scaffolds more closely resembling the scale and architecture of native ECM would lead to improved cellular responses. Previous studies have shown that scaffolds containing both micro and nanofibers result in better cellular adhesion, viability and scaffold infiltration as well as ECM production when compared to single-scaled fiber controls [10, 11, 13, 14, 34]. The benefit of multiscale fibrous scaffold generation by continuous means as investigated here over previously described methods, such as tandem melt deposition with electrospinning or layer-bylayer electrospinning, is that fabrication via the dual extrusion method with a rotating mandrel offers more reproducibility due to fewer steps and minimized human influence on fabrication. Also, scaffolds formed in this manner demonstrate a homogenous distribution of micro and nanofibers throughout the entire scaffold while other fabrication techniques often lead to stratified compositions. While Soliman et al showed that simultaneous dual extrusion was useful for the production of multiscale fibrous scaffolds, methods described in the current work demonstrate the added value of fabricating scaffolds 


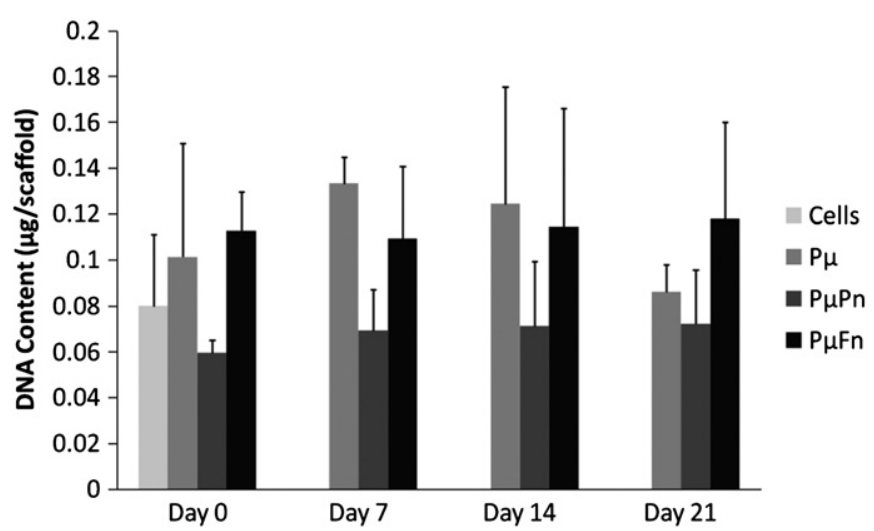

Figure 7. Scaffold cellularity as determined by total DNA content per scaffold. In this instance, the group indicated as 'cells' denotes the DNA content of the initial cell suspension used to seed each of the scaffold types on day 0 . DNA contents are represented as mean \pm standard deviation for $n=4$ samples.

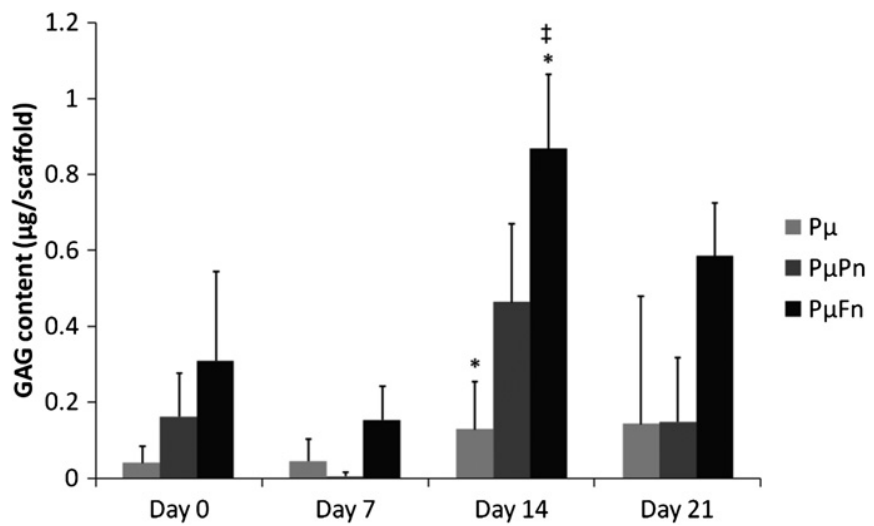

Figure 8. GAG content of scaffolds at days $0,7,14$, and 21 as determined by the DMMB assay. GAG contents are represented as mean \pm standard deviation for $n=4$ samples. Different scaffold types marked with an asterisk, '*', indicate significant difference in GAG content for a specific time point. Statistical significance of a particular scaffold type at a specific culture period compared to both of the other scaffold types at every other time point is indicated by a '十'.

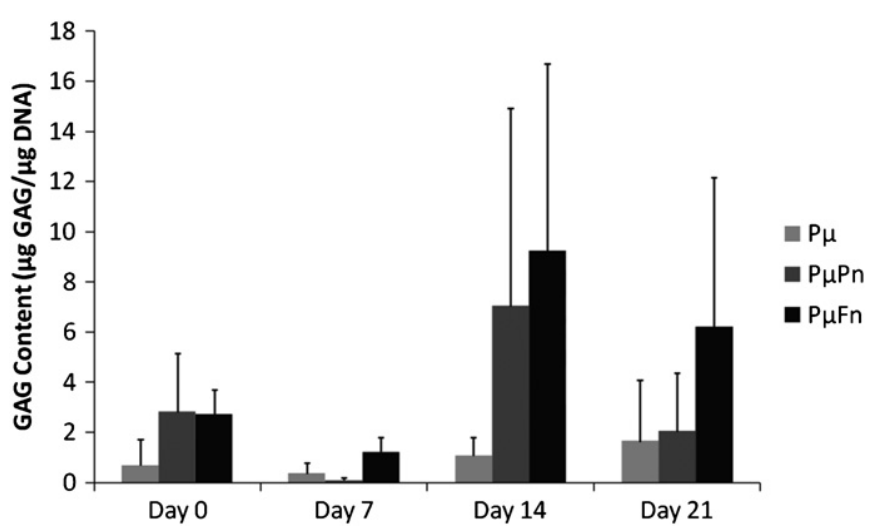

Figure 9. GAG content normalized by total DNA content per scaffold at days $0,7,14$ and 21 as determined by the DMMB assay. Normalized GAG contents are represented as mean \pm standard deviation for $n=4$ samples. with fibers composed of different materials in an uninterrupted fashion [14]. This additional feature increases the capacity of this system to fine-tune the generation of a particular scaffold for a specific application.

To examine the cellular response to nanofibers while trying to maintain larger pore sizes for cellular infiltration, different scaffolds were fabricated containing both micro and nanofibers. This was accomplished by extruding polymer solutions at different rates and applied voltages from opposite sides of a rotating mandrel. In this manner, scaffolds containing both micro and nanofibers as well as fibers of two different materials, PCL and fibrin, were generated. A higher voltage was needed to produce fibrin nanofibers in a reproducible manner than was needed for the production of PCL nanofibers, quite possibly due to a difference in viscosity, surface tension and/or conductivity of the polymer solutions used. As a result of this difference, the diameter of the microfibers within the $\mathrm{P} \mu \mathrm{Fn}$ scaffolds was significantly lower than that of the microfibers in the other two scaffold types. It is likely that the change in the electric field of the whole system was responsible for the observed slight decrease in PCL microfiber diameter for the $\mathrm{P} \mu \mathrm{Fn}$ scaffolds compared to the $\mathrm{P} \mu \mathrm{Pn}$ scaffolds. Images of vertical scaffold cross-sections demonstrated that this fabrication method was successful in generating scaffolds in which the different fiber types were well mixed throughout the entire scaffold (figures $2(g)$ (i)). The nanofiber containing scaffolds that were generated contained visibly different nanofiber densities with the $\mathrm{P} \mu \mathrm{Pn}$ scaffolds exhibiting more densely packed nanofibers. As a result, despite having comparable porosities, the $\mathrm{P} \mu \mathrm{Pn}$ scaffolds displayed smaller pore sizes than the $\mathrm{P} \mu \mathrm{Fn}$ scaffolds.

Confocal images taken shortly after cell attachment showed that hMSCs on scaffolds containing PCL nanofibers, $\mathrm{P} \mu \mathrm{Pn}$, were broad and flattened and appeared to span intermicrofiber gaps (figure 3). Likewise, SEM and histology images revealed that scaffolds containing PCL nanofibers exhibited a surface densely coated with cells and ECM. This is possibly due to the smaller pore sizes of the $\mathrm{P} \mu \mathrm{Pn}$ scaffolds as a result of dense nanofiber packing which could partially restrict cellular infiltration. Fast Green staining of histological sections supports this theory, showing that while the maximum cellular infiltration was similar for all three scaffold types, proportionally more of the cells in the $\mathrm{P} \mu \mathrm{Pn}$ scaffolds remained in the upper region of the scaffold. Compared to the $\mathrm{P} \mu \mathrm{Pn}$ scaffolds, the $\mathrm{P} \mu$ and $\mathrm{P} \mu \mathrm{Fn}$ scaffolds appeared to exhibit greater Fast Green staining at the ultimate depth of infiltration. The difference in cellular infiltration patterns of the $\mathrm{P} \mu \mathrm{Pn}$ scaffolds in comparison to the $\mathrm{P} \mu$ and $\mathrm{P} \mu \mathrm{Fn}$ scaffolds may be due to the relative pore sizes of the different scaffold types. Similarly, it is possible that the distribution of nanofibers with respect to microfibers within the $\mathrm{P} \mu \mathrm{Pn}$ scaffolds provided a method to assist cells in spanning inter-microfiber gaps. These cellular bridges in turn may have resulted in the dispersion of cells in the upper portion of the scaffold, while within this region $\mathrm{P} \mu$ and $\mathrm{P} \mu \mathrm{Fn}$ scaffolds displayed cells primarily on the scaffold surface.

Confocal images taken $3 \mathrm{~h}$ after initial cell attachment revealed that hMSCs on $\mathrm{P} \mu$ and $\mathrm{P} \mu \mathrm{Fn}$ scaffolds 
spread maintaining an elongated, spindle-like morphology characteristic of MSCs. On these two scaffolds, hMSCs primarily attached along the length of microfibers as seen in confocal and SEM images (figures 3 and 4). In contrast, cells attached to $\mathrm{P} \mu \mathrm{Pn}$ scaffolds spread appearing broad and flat spanning the space between microfibers. As such, the cells spread on the nanofibers of the $\mathrm{P} \mu \mathrm{Pn}$ scaffolds resembling two-dimensional cultures as opposed to the intended threedimensional scaffold environment. Furthermore, the broad and flat hMSC morphology could potentially have an adverse effect on tissue development within a scaffold as previous studies have shown that a flat polygonal cell shape sometimes precedes reduced proliferation and chondrogenic differentiation capacity compared to more elongated cells with spindle-like morphologies [35-37].

In a serum-free culture environment without additional growth factors, it is not uncommon to see limited cellular proliferation [6, 38]. Here, there was neither a significant increase nor decrease in total DNA content of all scaffold types over the course of 21 days. This indicated that all three scaffold types were sufficient for supporting MSC culture. Sometimes it is desirable to induce increased cellular proliferation as greater cell numbers may lead to increased ECM deposition. However, as cells proliferate metabolic resources are somewhat allocated away from matrix production. In this fashion, when attempting to generate ample ECM within a polymeric scaffold, it is important to not only consider the effect of the scaffold on cellular proliferation but also on the ECM content of the scaffolds as culture progresses. As such in addition to quantifying the DNA content of the different scaffold types over time, the GAG content of the scaffolds was also measured. From the DMMB biochemical assay, it was determined that PCL microfibers interspersed with fibrin nanofibers exhibited significantly higher GAG contents at day 14 than the PCL microfiber control group. Furthermore, at day 14 the $\mathrm{P} \mu \mathrm{Fn}$ samples contained more GAG total than both of the other scaffold types at the various time points with exception to the $\mathrm{P} \mu \mathrm{Pn}$ samples on day 14 . These results suggest that the presence of nanofibers, specifically fibrin nanofibers, within the scaffold had a positive influence on the production of GAGs by hMSCs in 14 days of culture even in the absence of growth factors. It was hypothesized that the inclusion of nanofibers would lead to a significant increase in GAG production by the differentiating cells because nanoscale features more closely resemble the components of native ECM and as such may lead to improved cellular responses [5, 6]. However, both the total GAG content of the scaffolds and GAG/DNA amounts stayed relatively constant throughout the entire culture duration. One possible explanation for this is that some soluble GAGs produced by the cells may have diffused into the surrounding culture medium rather than remaining within the scaffold. Evidence of this behavior has been shown in previous studies measuring GAG content as a diagnostic of tissue engineered cartilage development as well as cartilage explant culture [33, 39-41].

Histological sections exhibited increased proteoglycan deposits with the incorporation of nanofibers within microfiber meshes as indicated by Safranin $\mathrm{O}$ staining. This suggests that the cells responded to the nanofibers in the scaffolds by producing more ECM as compared to scaffolds composed of microfibers alone. This evidence supports the hypothesis that a scaffold architecture more closely resembling a native ECM, whether it is with respect to scale or composition, could be advantageous for cartilage-like matrix production and deposition.

While it is difficult to make direct comparisons between scaffolds that have been fabricated using different extrusion rates and post-fabrication processing techniques, this work does show that it is feasible to produce biodegradable nonwoven fibrous meshes composed of microfibers mixed with nanofibers throughout an entire scaffold. Also, it is possible to produce such multiscale scaffolds containing fibers of two discrete compositions. One of the limitations of the electrospinning technique is that processing parameters such as the extrusion flow rate and voltage are dependent on the polymer solution composition. Due to the processing limitations necessary to produce nanofibers from distinct compositions, it is necessary to further examine the effect extrusion rates have on scaffold characteristics and cellular responses. Cell culture on these multiscale scaffolds has shown that the inclusion of nanofibers does not negatively affect cell growth or matrix production. In fact, histological images suggest that the inclusion of nanofibers is beneficial toward the production and distribution of GAGs. The interaction between fiber composition, scale, porosity and pore size as related to the development of the ECM should be more closely studied. Future work should look to fine-tune the density of nanofibers with respect to microfibers in an effort to harness the positive influence of nanofibers on cellular responses, such as improved ECM production and cell attachment, while minimizing any negative effects such as limited infiltration.

\section{Acknowledgments}

The authors appreciate the Indo-US Science and Technology Forum under the Joint Centre on 'Cell Targeted Diagnostics and Therapy Using Nanomaterials' for supporting a collaborative research exchange facilitating cooperative research efforts between international institutions. The authors are also thankful to support of the Nanomission, Department of Science and Technology, the Government of India, under a grant for theragnostics and regenerative engineering using nanostructured materials. Efforts toward developing multiscale fibrous scaffolds for tissue engineering applications have been supported from funding by the National Institutes of Health (R01 AR57083). Furthermore, EJL is grateful for assistance from the Rice Institute of Biosciences and Bioengineering's Biotechnology Training grant (NIH T32 GM08362).

\section{References}

[1] Lowery J L, Datta N and Rutledge G C 2010 Effect of fiber diameter, pore size and seeding method on growth of human dermal fibroblasts in electrospun poly( $\varepsilon$-caprolactone) fibrous mats Biomaterials 31 491-504 
[2] Alvarez-Barreto J, Linehan S, Shambaugh R and Sikavitsas V 2007 Flow perfusion improves seeding of tissue engineering scaffolds with different architectures Ann. Biomed. Eng. 35429

[3] Gomes M E, Holtorf H L, Reis R L and Mikos A G 2006 Influence of the porosity of starch-based fiber mesh scaffolds on the proliferation and osteogenic differentiation of bone marrow stromal cells cultured in a flow perfusion bioreactor Tissue Eng. 12 801-9

[4] Li W-J, Laurencin C T, Caterson E J, Tuan R S and Ko F K 2002 Electrospun nanofibrous structure: a novel scaffold for tissue engineering J. Biomed. Mater. Res. 60 613-21

[5] Li W-J, Tuli R, Huang X, Laquerriere P and Tuan RS 2005 Multilineage differentiation of human mesenchymal stem cells in a three-dimensional nanofibrous scaffold Biomaterials 265158

[6] Li W-J, Danielson K G, Alexander P G and Tuan R S 2003 Biological response of chondrocytes cultured in three-dimensional nanofibrous poly ( $\varepsilon$-caprolactone) scaffolds J. Biomed. Mater. Res. A 67 1105-14

[7] Pham Q P, Sharma U and Mikos A G 2006 Electrospun $\operatorname{poly}(\varepsilon$-caprolactone) microfiber and multilayer nanofiber/microfiber scaffolds: characterization of scaffolds and measurement of cellular infiltration Biomacromolecules 7 2796-805

[8] Shalumon K T, Binulal N S, Deepthy M, Jayakumar R, Manzoor K and Nair S V 2011 Preparation, characterization and cell attachment studies of electrospun multi-scale poly(caprolactone) fibrous scaffolds for tissue engineering J. Macromol. Sci. A 48 21-30

[9] Srinivasan S, Jayakumar R, Chennazhi K P, Levorson E J, Mikos A G and Nair S V 2012 Multiscale fibrous scaffolds in regenerative medicine Adv. Polym. Sci. 246 1-20

[10] Park S H, Kim T G, Kim H C, Yang D-Y and Park T G 2008 Development of dual scale scaffolds via direct polymer melt deposition and electrospinning for applications in tissue regeneration Acta Biomaterialia 4 1198-207

[11] Tuzlakoglu K, Bolgen N, Salgado A, Gomes M, Piskin E and Reis R 2005 Nano- and micro-fiber combined scaffolds: a new architecture for bone tissue engineering J. Mater. Sci., Mater. Med. 16 1099-104

[12] Martins A, Pinho E D, Correlo V M, Faria S, Marques A P, Reis R L and Neves N M 2010 Biodegradable nanofibers-reinforced microfibrous composite scaffolds for bone tissue engineering Tissue Eng. A 16 3599-609

[13] Mota C, Puppi D, Dinucci D, Errico C, Bártolo P and Chiellini F 2011 Dual-scale polymeric constructs as scaffolds for tissue engineering Materials 4 527-42

[14] Soliman S et al 2010 Multiscale three-dimensional scaffolds for soft tissue engineering via multimodal electrospinning Acta Biomaterialia 6 1227-37

[15] Chen W-C, Yao C-L, Wei Y-H and Chu IM 2011 Evaluating osteochondral defect repair potential of autologous rabbit bone marrow cells on type II collagen scaffold Cytotechnology 63 13-23

[16] Kawasaki K, Ochi M, Uchio Y, Adachi N and Matsusaki M 1999 Hyaluronic acid enhances proliferation and chondroitin sulfate synthesis in cultured chondrocytes embedded in collagen gels J. Cell. Physiol. 179 142-8

[17] Im G-I 2005 Chondrogenesis from mesenchymal stem cells derived from adipose tissue on the fibrin scaffold Curr. Appl. Phys. 5 438-43

[18] Matthews J A, Boland E D, Wnek G E, Simpson D G and Bowlin G L 2003 Electrospinning of collagen type II: a feasibility study J. Bioact. Compatible Polym. 18 125-34

[19] Duggal S, Frønsdal K B, Szöke K, Shahdadfar A, Melvik J E and Brinchmann J E 2009 Phenotype and gene expression of human mesenchymal stem cells in alginate scaffolds Tissue Eng. A 15 1763-73
[20] Vinatier C, Mrugala D, Jorgensen C, Guicheux J and Noël D 2009 Cartilage engineering: a crucial combination of cells, biomaterials and biofactors Trends Biotechnol. 27 307-14

[21] Kavalkovich K W, Boynton R E, Murphy J M and Barry F 2002 Chondrogenic differentiation of human mesenchymal stem cells within an alginate layer culture system In Vitro Cell. Dev. Biol. Anim. 38 457-66

[22] Ma H L, Hung S C, Lin S Y, Chen Y L and Lo W H 2003 Chondrogenesis of human mesenchymal stem cells encapsulated in alginate beads J. Biomed. Mater. Res. A $64273-81$

[23] Medrado G C B, Machado C B, Valerio P, Sanches M D and Goes A M 2006 The effect of a chitosan-gelatin matrix and dexamethasone on the behavior of rabbit mesenchymal stem cells Biomed. Mater. 1 155-61

[24] Geng X, Kwon O-H and Jang J 2005 Electrospinning of chitosan dissolved in concentrated acetic acid solution Biomaterials 26 5427-32

[25] Perumcherry S R, Chennazhi K P, Nair S V, Menon D and Afeesh R 2011 A novel method for the fabrication of fibrin-based electrospun nanofibrous scaffold for tissue-engineering applications Tissue Eng. C 17 1121-30

[26] Blomback B, Blomback M, Edman P and Hessel B 1966 Human fibrinopeptides. Isolation, characterization and structure Biochim. Biophys. Acta 115 371-96

[27] Cavallo C et al 2011 Comparison of alternative mesenchymal stem cell sources for cell banking and musculoskeletal advanced therapies J. Cell. Biochem. 112 1418-30

[28] Capelli C et al 2011 Minimally manipulated whole human umbilical cord is a rich source of clinical-grade human mesenchymal stromal cells expanded in human platelet lysate Cytotherapy 13 786-801

[29] Kern S, Eichler H, Stoeve J, Klüter H and Bieback K 2006 Comparative analysis of mesenchymal stem cells from bone marrow, umbilical cord blood, or adipose tissue Stem Cells 24 1294-301

[30] Lee O K, Kuo T K, Chen W-M, Lee K-D, Hsieh S-L and Chen T-H 2004 Isolation of multipotent mesenchymal stem cells from umbilical cord blood Blood 103 1669-75

[31] Ramis J M, Rubert M, Vondrasek J, Gayà A, Lyngstadaas S P and Monjo M 2012 Effect of enamel matrix derivative and of proline-rich synthetic peptides on the differentiation of human mesenchymal stem cells toward the osteogenic lineage Tissue Eng. A 18 1253-63

[32] Nitya G, Nair GT, Mony U, Chennazhi K P and Nair S V 2012 In vitro evaluation of electrospun $\mathrm{pcl} /$ nanoclay composite scaffold for bone tissue engineering J. Mater. Sci.,: Mater. Med. 23 1749-61

[33] Liao J, Guo X, Grande-Allen K J, Kasper F K and Mikos A G 2010 Bioactive polymer/extracellular matrix scaffolds fabricated with a flow perfusion bioreactor for cartilage tissue engineering Biomaterials 31 8911-20

[34] Kim S J, Jang D H, Park W H and Min B-M 2010 Fabrication and characterization of 3-dimensional PLGA nanofiber/microfiber composite scaffolds Polymer 51 1320-7

[35] Seeberger K L, Dufour J M, Shapiro A M J, Lakey J R T, Rajotte R V and Korbutt G S 2006 Expansion of mesenchymal stem cells from human pancreatic ductal epithelium Lab. Invest. 86 141-53

[36] Mets T and Verdonk G 1981 In vitro aging of human bone marrow derived stromal cells Mech. Ageing Dev. 16 81-9

[37] Neuhuber B, Swanger S A, Howard L, Mackay A and Fischer I 2008 Effects of plating density and culture time on bone marrow stromal cell characteristics Exp. Hematol. 36 1176-85

[38] Tonti G A and Mannello F 2008 From bone marrow to therapeutic applications: different behaviour and genetic/epigenetic stability during mesenchymal stem cell 
expansion in autologous and foetal bovine sera? Int. J. Dev. Biol. 52 1023-32

[39] Mahmood T A, Shastri V P, Van Blitterswijk C A, Langer R and Riesle $\mathrm{J} 2005$ Tissue engineering of bovine articular cartilage within porous poly(ether ester) copolymer scaffolds with different structures Tissue Eng. 11 1244-53
[40] Wang L, Seshareddy K, Weiss M L and Detamore M S 2009 Effect of initial seeding density on human umbilical cord mesenchymal stromal cells for fibrocartilage tissue engineering Tissue Eng. A 15 1009-17

[41] Bolis S, Handley C J and Comper W D 1989 Passive loss of proteoglycan from articular cartilage explants Biochim. Biophys. Acta 993 157-67 